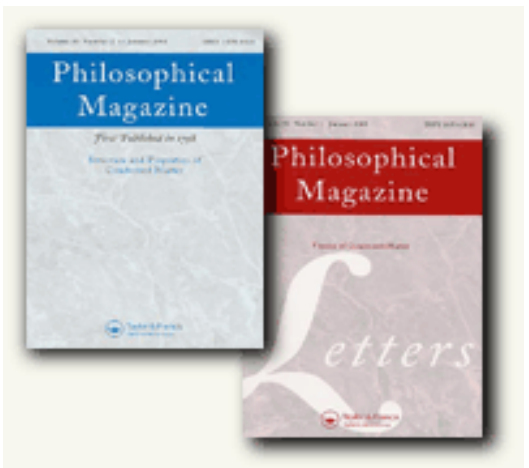

\title{
Simulation of the Dynamics of Hard Ellipsoids
}

\begin{tabular}{|r|l|}
\hline Journal: & Philosophical Magazine \& Philosophical Magazine Letters \\
\hline Manuscript ID: & TPHM-08-May-0195.R1 \\
\hline Journal Selection: & Philosophical Magazine \\
\hline Date Submitted by the & 03-Oct-2008 \\
\hline $\begin{array}{r}\text { Complete List of Authors: } \\
\text { Keywords: }\end{array}$ & $\begin{array}{l}\text { De Michele, Cristiano; University of Roma, Physics } \\
\text { Sciortino, Francesco; University of Rome, Physics } \\
\text { Schilling, Rolf; Johannes-Gutenberg-Universitat Mainz, Physics }\end{array}$ \\
\hline $\begin{array}{l}\text { Keywords (user supplied): } \\
\text { glass transition, molecular, molecular dynamics }\end{array}$ \\
\hline $\begin{array}{l}\text { Note: The following files were submitted by the author for peer review, but cannot be converted liquids } \\
\text { to PDF. You must view these files (e.g. movies) online. }\end{array}$ \\
\hline $\begin{array}{l}\text { DeMichele.tex } \\
\text { DeMichele.bib } \\
\text { DeMichele.bbl }\end{array}$ \\
\hline
\end{tabular}

\section{s ScholarONE" \\ Manuscript Central}




\section{Introduction}

Even if particles interact with only excluded volume interactions, they may exhibit a rich phase diagram, for example it is known that simple non-spherical hard-core particles can form either crystalline or liquid crystalline ordered phases [1], as first shown analytically by Onsager [2] for rod-like particles. Accurate phase diagrams of several hard-body shapes can be found in literature [3-6] but detailed informations about dynamics properties and kinetically arrested states of hard-core bodies are not available. While mode coupling theory (MCT) [7] well describes the slowing down of the dynamics of hard-sphere system on increasing the packing fraction $\phi$, its molecular counterpart, the molecular model coupling theory (MMCT) [8] has not been tested in simulated or real systems yet. On going from spheres to non-spherical particles, non-trivial phenomena arise, due to the interplay between translational and rotational degrees of freedom. The slowing down of the dynamics can indeed appear either in both translational and rotational properties or in just one of the two. Hard ellipsoids (HE) of revolution [1, 9] are one of the most prominent systems composed by hard body anisotropic particles and MMCT has

\footnotetext{
*Corresponding author. Email: cristiano.demichele@roma1.infn.it

${ }^{1} X_{0}=a / b$, where $a$ is the length of the revolution axis and $b$ is the length of the two other axes. 
been applied to HE $[10,11]$, predicting a swallow-like glass transition line. In addition, the theory suggests that for $X_{0} \lesssim 0.5$ and $X_{0} \gtrsim 2$, the glass transition is driven by a precursor of nematic order, resulting in an orientational glass where the translational density fluctuations are quasi-ergodic, except for very small wave vectors $q$.

\section{Methods}

We perform an extended study of the dynamics of monodisperse HE in a wide window of $\phi$ and $X_{0}$ values, extending the range of $X_{0}$ previously studied [12].

We study the translational and rotational mean squared displacements, looking for evidences of cage effect and the translational and rotational correlation functions, to search for the onset of slowing down and stretching in the decay of the correlation. We also focus on establishing the trends leading to dynamic slowing down in both translations and rotations, by evaluating the loci of constant translational and rotational diffusion. These lines, in the limit of vanishing diffusivities, approach the glass-transition lines. We simulate a system of $N=512$ ellipsoids at various volumes $V=L^{3}$ in a cubic box of edge $L$ with periodic boundary conditions. We chose the geometric mean of the axis $l=\sqrt[3]{a b^{2}}$ as unit of distance, the mass $m$ of the particle as unit of mass $\left(m=1\right.$ ) and $k_{B} T=1$ (where $k_{B}$ is the Boltzmann constant and $T$ is the temperature) and hence the corresponding unit of time is $\sqrt{m l^{2} / k_{B} T}$. The inertia tensor is chosen as $I_{x}=I_{y}=2 m r^{2} / 5$, where $r=\min \{a, b\}$. The value of the $I_{z}$ component is irrelevant [13], since the angular velocity along the symmetry (z-) axis of the HE is conserved. We simulate a grid of more than 500 state points at different $X_{0}$ and $\phi$. To create the starting configuration at a desired $\phi$, we generate a random distribution of ellipsoids at very low $\phi$ and then we progressively decrease $L$ up to the desired $\phi$. We then equilibrate the configuration by propagating the trajectory for times such that both angular and translational correlation functions have decayed to zero. Finally, we perform a production run at least 30 times longer than the time needed to equilibrate. For the points close to the isotropic-nematic (I-N) transition we check the nematic order by evaluating the largest eigenvalue $S$ of the order tensor (for further details see $[14])$.

\section{Results and discussion}

\subsection{Mean squared displacements}

A first characterization of HE dynamics is offered by translational and rotational mean squared displacements (MSD). Translational MSD is defined as:

$$
\left\langle r^{2}(t)\right\rangle=\frac{1}{N} \sum_{i}\left\langle\left\|\mathbf{x}_{i}(t)-\mathbf{x}_{i}(0)\right\|^{2}\right\rangle
$$

where $N$ is the number of $\mathrm{HE}$ and $\mathbf{x}_{i}(t)$ is the position of the center-of-mass of the $i$-th HE. Analogously the rotational MSD is:

$$
\left\langle\Phi^{2}(t)\right\rangle=\frac{1}{N} \sum_{i}\left\langle\left\|\Delta \Phi_{i}\right\|^{2}\right\rangle
$$

where $\Delta \Phi_{i}=\int_{0}^{t} \omega_{i} d t, \omega_{i}$ being the angular velocity of the $i$-th HE. In Fig. 2 trans- 


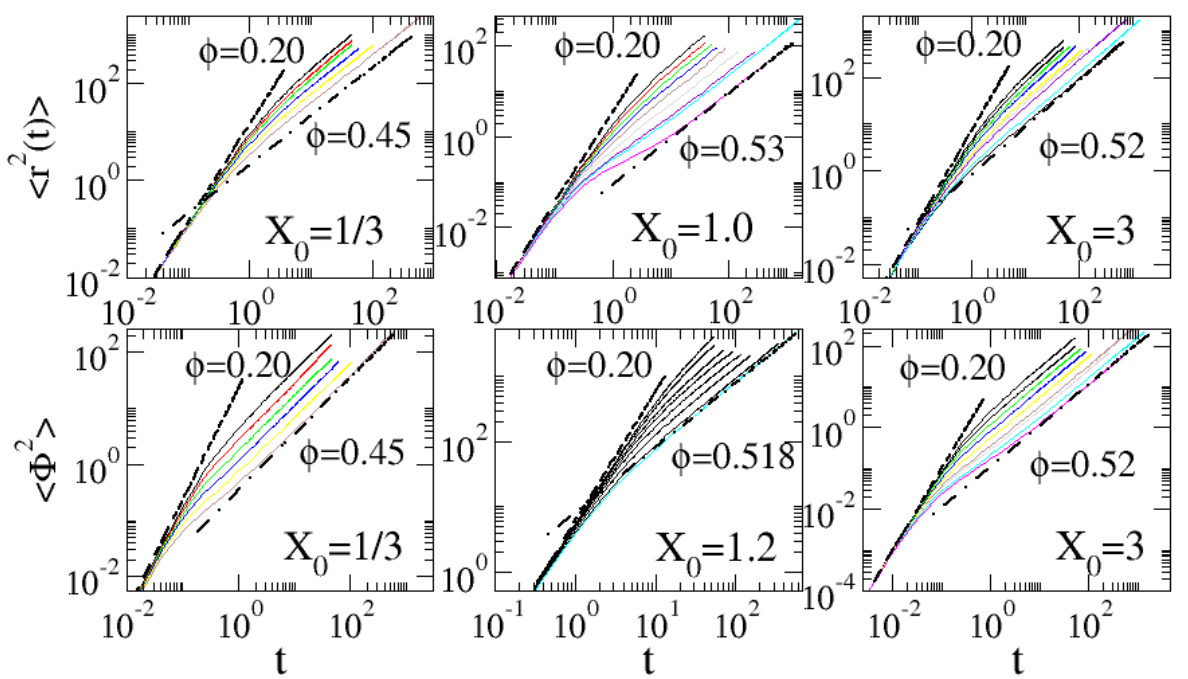

Figure 1. [Color online]Translational (top) and rotational (bottom) MSD for three different elongations at different volume fractions. Top (from left to right) $X_{0}=1 / 3$ (from top to bottom: $\phi=0.20 \ldots 0.45$ ), 1.0 $(\phi=0.20 \ldots 0.53), 3.0(\phi=0.20 \ldots 0.52)$. Bottom (from left to right): $X_{0}=1 / 3(\phi=0.20 \ldots 0.45), 1.2$ $(\phi=0.20 \ldots 0.518), 3.0(\phi=0.20 \ldots 0.52)$. Dashed lines and dot-dashed lines are guides to eye, showing the ballistic and diffusive regime respectively.
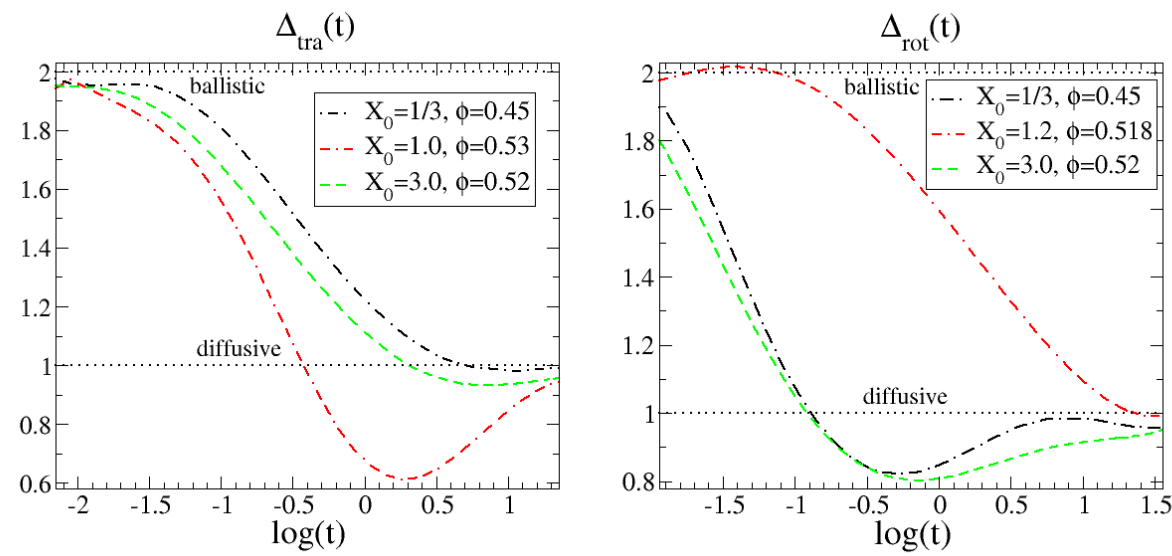

Figure 2. [Color online] $\Delta_{t r a}(t)$ and $\Delta_{r o t}(t)$ (see text for their definitions) for the most "supercooled" state points simulated for four different elongations. Dotted lines indicate ballistic and diffusive regimes.

lational and rotational MSD are reported for three different elongations at different volume fractions. Both translational and rotational MSD shows for short times a ballistic regime, where $\left\langle r^{2}(t)\right\rangle,\left\langle\Phi^{2}(t)\right\rangle \propto t^{2}$ that crosses over to a diffusion process at long times, i.e. $\left\langle r^{2}(t)\right\rangle,\left\langle\Phi^{2}(t)\right\rangle \propto t$. For the state points simulated in the present study, for $\left\langle\Phi^{2}\right\rangle$ the ballistic short-time and the diffusive long-time regimes separated by an intermediate time windows, due to particles caging, only for high elongations (i.e. for $X_{0}=1 / 3$ and $X_{0}=3$ in Fig. 2. In contrast for $\left\langle r^{2}\right\rangle$ this intermediate regime shows up only for elongations close to 1 . The scenario just depicted is more apparent if we consider the quantities: $\Delta_{t r a}(t)=\partial \log \left(\left\langle r^{2}(t)\right\rangle\right) / \partial \log t$ and $\Delta_{\text {rot }}(t)=\partial \log \left(\left\langle\Phi^{2}(t)\right\rangle\right) / \partial \log t$. The short- and the long-time limits of $\Delta_{\text {tra }}(t)$, $\Delta_{\text {rot }}(t)$ correspond to the ballistic ( $\left.\Delta_{\text {tra }}(0), \Delta_{\text {rot }}(0)=2\right)$ and the diffusive regimes 


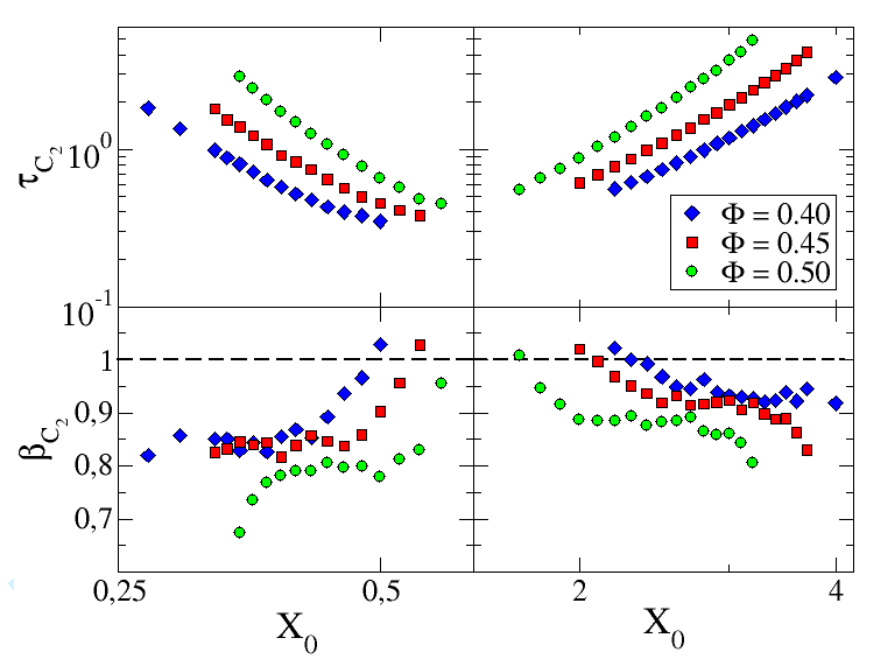

Figure 3. [Color online] $\beta_{C_{2}}$ and $\tau_{C_{2}}$ are obtained from fits of $C_{2}$ to a stretched exponential for $\phi=$ $0.40,0.45$ and 0.50 . Top: $\tau_{C_{2}}$ as a function of $X_{0}$. Bottom: $\beta_{C_{2}}$ as a function of $X_{0}$. The time window used for the fits is chosen in such a way to exclude the microscopic short times ballistic relaxation. For $0.588<X_{0}<1.7$ the orientational relaxation is exponential.

$\left(\Delta_{\text {tra }}(\infty), \Delta_{\text {rot }}(\infty)=1\right)$, respectively. Anyway, if the translational, or rotational, velocity autocorrelation function ( $\mathrm{VCF}$ ) exhibits a negative tail at long times, $\Delta_{\text {tra }}(t)$ shows a minimum. In other words a monotonically decreasing translational (rotational) $\mathrm{VCF}$, that is without cage effect, corresponds to a monotonically decreasing $\Delta_{\text {tra }}(t)\left(\Delta_{\text {rot }}(t)\right)[15]$.

\subsection{Orientational correlation function}

Cage effect of $\left\langle\Phi^{2}(t)\right\rangle$ for large $X_{0}$, approaching the nematic phase, supports the possibility of a close-by glass transition. To further support this possibility we evaluate the self part of the intermediate scattering function $F_{\text {self }}(q, t)=$ $\frac{1}{N}\left\langle\sum_{j} e^{i \mathbf{q} \cdot\left(\mathbf{x}_{j}(t)-\mathbf{x}_{j}(0)\right)}\right\rangle$ and the second order orientational correlation function $C_{2}(t)$ defined as [12] $C_{2}(t)=\left\langle P_{2}(\cos \theta(t))\right\rangle$, where $P_{2}(x)=\left(3 x^{2}-1\right) / 2$ and $\theta(t)$ is the angle between the symmetry axis at time $t$ and at time 0 . The over-compressing achievable for HE is rather limited (as for the well known hard-sphere case), nevertheless rotational and translational correlation functions reveals that the onset of dynamic slowing down and glassy dynamics can be detected by the appearance of stretching. $F_{\text {self }}$ shows an exponential behaviour close to the I-N transition $\left(X_{0}=3.2,0.3448\right)$ on the prolate and oblate side and only when $X_{0} \approx 1, F_{\text {self }}$ develops a small stretching, consistent with the minimum of the swallow-like curve observed in the fluid-crystal line $[16,17]$, in the jamming locus as well as in the predicted behavior of the glass line for $\mathrm{HE}[8]$ and for small elongation dumbbells $[18,19]$.

In contrast to the $F_{\text {self }}$ behavior, the orientational correlation function $C_{2}$ shows stretching at large anisotropy, i.e. at small and large $X_{0}$ values, but decays within the microscopic time for almost spherical particles, in accordance with the cage effect of $\left\langle\Phi^{2}(t)\right\rangle$. Previous studies of the rotational dynamics of HE [12] did not report stretching in $C_{2}$, probably due to the smaller values of $X_{0}$ previously investigated and to the present increased statistic which allows us to follow the full decay of the correlation functions.

In summary $C_{2}$ becomes stretched approaching the I-N transition while $F_{\text {self }}$ 


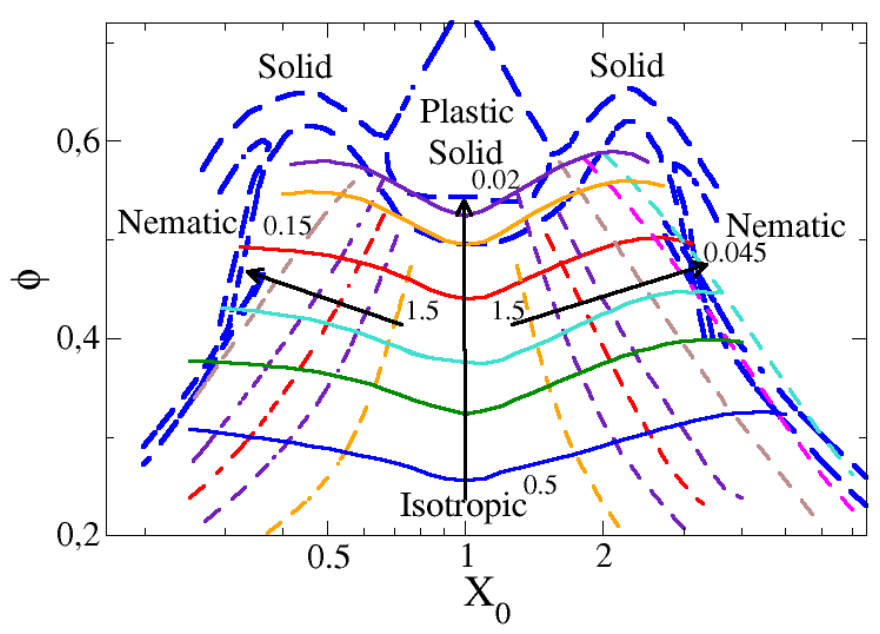

Figure 4. [Color online] Isodiffusivity lines. Solid lines are isodiffusivity lines from translational diffusion coefficients $D_{\text {trans }}$ and dashed lines are isodiffusivities lines from rotational diffusion coefficients $D_{\text {rot }}$. Arrows indicate decreasing diffusivities. Left and right arrows refer to rotational diffusion coefficients. Diffusivities along left arrow are: $1.5,0.75,0.45,0.3,0.15$. Diffusivities along right arrow are: $1.5,0.75$, $0.45,0.3,0.15,0.075,0.045$. Central arrow refers to translational diffusion coefficients, whose values are: 0.5 , $0.3,0.2,0.1,0.04,0.02$. Thick Long-dashed curves are coexistence curves of all first order phase transitions in the phase diagram of HE evaluated by Frenkel and Mulder[20] Solid lines are coexistence curves for the I-N transition of oblate and prolate ellipsoids, obtained analytically by Tijpto-Margo and Evans [6].

remains exponential on approaching the transition. To quantify the amount of stretching in $C_{2}$, we fit it to the function $A \exp \left[-\left(t / \tau_{C_{2}}\right)^{\beta_{C_{2}}}\right]$ (stretched exponential) for several state points and we show in Fig. 3 the $X_{0}$ dependence of $\tau_{C_{2}}$ and $\beta_{C_{2}}$ for three different values of $\phi$. In all cases, slowing down of the characteristic time and stretching increases progressively on approaching the I-N transition.

\subsection{Isodiffusivity lines}

For all the simulated state points, we evaluated the translational $\left(D_{\text {trans }}\right)$ and rotational $\left(D_{\text {rot }}\right)$ diffusion coefficients. Then by proper interpolation of $D_{\text {trans }}$ and $D_{\text {rot }}$, we evaluate the corresponding isodiffusivity lines, that are the loci of points, in the $X_{0}-\phi$ diagram, having the same diffusivity. Isodiffusivity lines are shown in Fig. 4. What emerges clearly from this figure is a striking decoupling of the translational and rotational dynamics: translational isodiffusivity lines resemble the swallow-like shape of the coexistence between the isotropic liquid and crystalline phases (as well as the MMCT prediction for the glass transition [8]), while rotational isodiffusivity lines have qualitatively the same shape of the I-N coexistence.

Translation isodiffusivity lines run almost parallel to $x$-axis, i.e. translational diffusion is mainly controlled by volume fraction (packing), that is the $y$-axis in Fig. 4. In contrast due to the almost perpendicular crossing of translational and rotational isodiffusivity lines, the rotational isodiffusivity lines are instead mostly controlled by $X_{0}$, showing a progressive slowing down of the rotational dynamics independently from the translational behavior. In other words moving along a translational isodiffusivity path, $D_{\text {rot }}$ progressively decreases, untilmkdir the rotational diffusion is completely arrested. Unfortunately in our specific case of monodisperse $\mathrm{HE}$, before reaching this point, the I-N nematic instability intervenes, and we were able to observe only a limited degree of supercooling. It would be intriguing to design a system of hard particles, where the nematic transition is completely inhibited. Likely this system can be obtained by a proper choice of the disorder in 
the particle's shape and/or elongations. MMCT predicts a nematic glass for large $X_{0}$ HE [8], in which orientational degrees of freedom start to freeze approaching the isotropic-nematic transition line, while translational degrees of freedom mostly remain ergodic. Hence our slowing down of the rotational dynamics is consistent with the results of this theory.

\section{Conclusions}

In summary we investigated the dynamics properties of a system of monodisperse $\mathrm{HE}$ and we have shown that clear precursors of dynamic slowing down, like the stretching of correlation functions and the cage effect, can be observed in the region of the phase diagram where a (meta)stable isotropic phase can be studied. In particular our data suggest, in accordance with MMCT predictions [8], at least two possible glass transition mechanisms: a slowing down in the orientational degrees of freedom (when $X_{0} \lesssim 0.5, X_{0} \gtrsim 2$ ), driven by the elongation of the particles and related to pre-nematic order (quantitative predictions about precursors effects of I-N transition can be also found in [21] and they can be checked using our data, work on this is under way), and a slowing down in the translational degrees of freedom (active for $0.5 \lesssim X_{0} \lesssim 2$ ) driven by packing and related to cage effect. The main effect of the existence of these two complementary arrest mechanisms is a decoupling of the translational and rotational dynamics which generates an almost perpendicular crossing of the $D_{\text {trans }}$ and $D_{\text {rot }}$ isodiffusivity lines.

\section{Acknowledgment}

We acknowledge support from MIUR-PRIN.

\section{References}

[1] M.P. Allen, Liquid Crystal Systems, N. Attig, K. Binder, H. Grubmüller and K. Kremer, eds., , Vol. 23, Jülich John von Neumann Institute for Computing, 2004, pp. 289-320.

[2] L. Onsager, Ann. N. Y. Acad. Sci. 51 (1949), pp. 627-659.

[3] J.D. Parsons, Phys. Rev. A 19 (1979), pp. 1225-1230.

[4] S.D. Lee, J. Chem. Phys. 89 (1989), pp. 7036-7037.

[5] A. Samborski, G.T. Evans, C.P. Mason, and M.P. Allen, Mol. Phys. 81 (1994), pp. 263-276.

[6] B. Tijpto-Margo and G.T. Evans, J. Chem. Phys. 93 (1990), pp. 4254-4265.

[7] W. Götze, Aspects of Structural Glass Transition, J.P. Hansen, D. Levesque and J. Zinn-Justin, eds., Amsterdam North-Holland, 1991, pp. 287-499.

[8] M. Letz, R. Schilling, and A. Latz, Ideal glass transition for hard ellipsoids, Phys. Rev. E 62 (2000), pp. $5173-5178$.

[9] G.S. Singh and B. Kumar, Ann. Phys. 294 (2001), pp. $24-47$.

[10] T. Franosch, M. Fuchs, W. Götze, M.R. Mayr, and A.P. Singh, Phys. Rev. E 56 (1997), pp. 5659-5674.

[11] R. Schilling and T. Scheidsteger, Phys. Rev. E 56 (1997), pp. 2932-2949.

[12] M.P. Allen and D. Frenkel, Phys. Rev. Lett. 58 (1987), pp. 1748-1750.

[13] M.P. Allen, D. Frenkel, and J. Talbot Molecular Dynamics Simulations Using Hard Particles, NorthHolland, 1989.

[14] C. De Michele, R. Schilling, and F. Sciortino, Dynamics of uniaxial hard ellipsoids, Phys. Rev. Lett. 98 (2007), pp. 265702-1-265702-4.

[15] L. Larini, A. Ottochian, C. De Michele, and D. Leporini, Universal scaling between structural relaxation and vibrational dynamics in glass-forming liquids and polymers, Nature Physics 4 (2008), pp. $42-45$.

[16] P.N. Pusey and W. Megenvan, Phys. Rev. Lett. 59 (1987), pp. 2083-2086.

[17] W.G. Hoover and F.H. Ree, J. Chem. Phys 49 (1968), pp. 3609-3617.

[18] S.H. Chong and W. Götze, Phys. Rev. E 65 (2002), pp. 041503-1-041503-17.

[19] S.H. Chong, A.J. Moreno, F. Sciortino, and W. Kob, Phys. Rev. Lett. 94 (2005), pp. 215701-1215701-4.

[20] D. Frenkel and B.M. Mulder, The hard ellipsoid-of-revolution fuid I. Monte Carlo simulations, Molec. Phys. 55 (1991), pp. 1171-1192.

[21] D. Kivelson and T. Keyes, Unified Theory of Orientational Relaxation, J. Chem. Phys. 57 (1972), pp. $4599-4612$. 


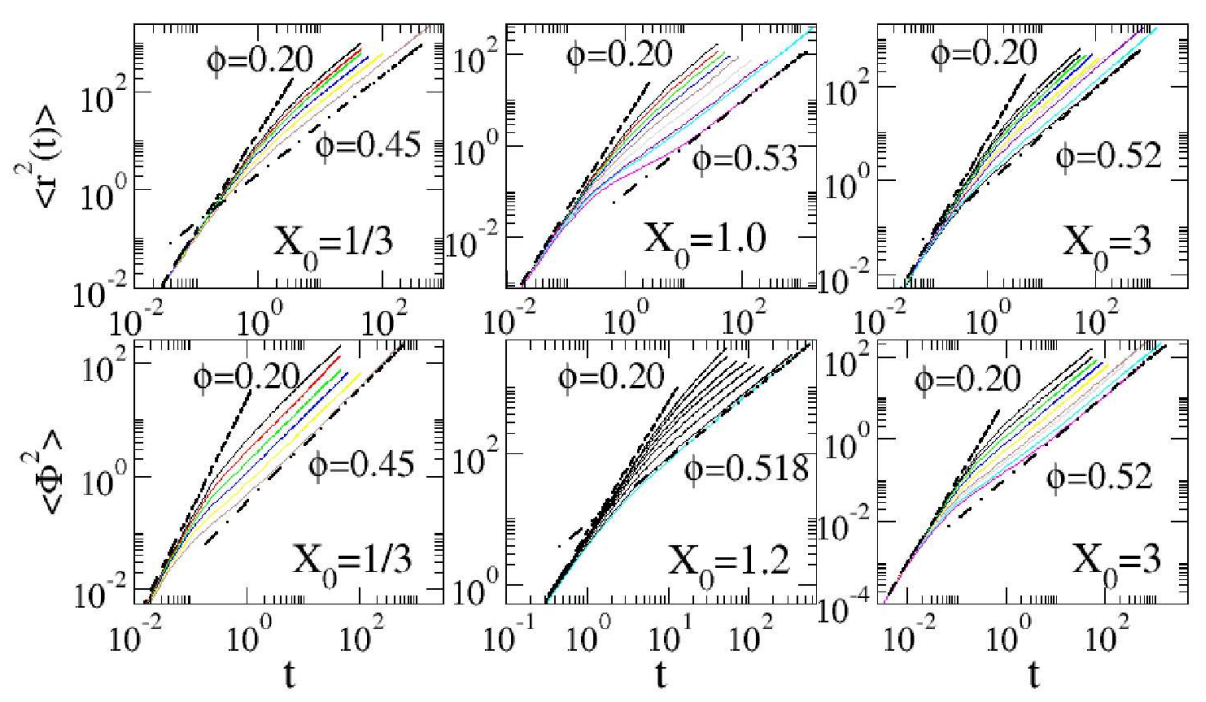

$338 \times 215 \mathrm{~mm}(600 \times 600 \mathrm{DPI})$ 

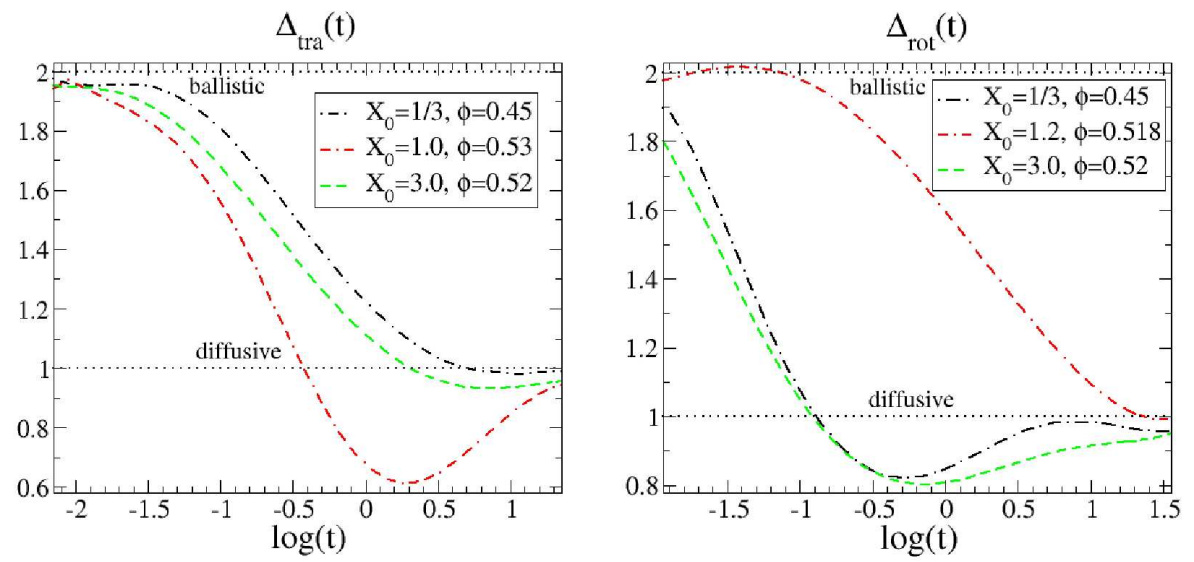

$457 \times 215 \mathrm{~mm}(600 \times 600 \mathrm{DPI})$ 


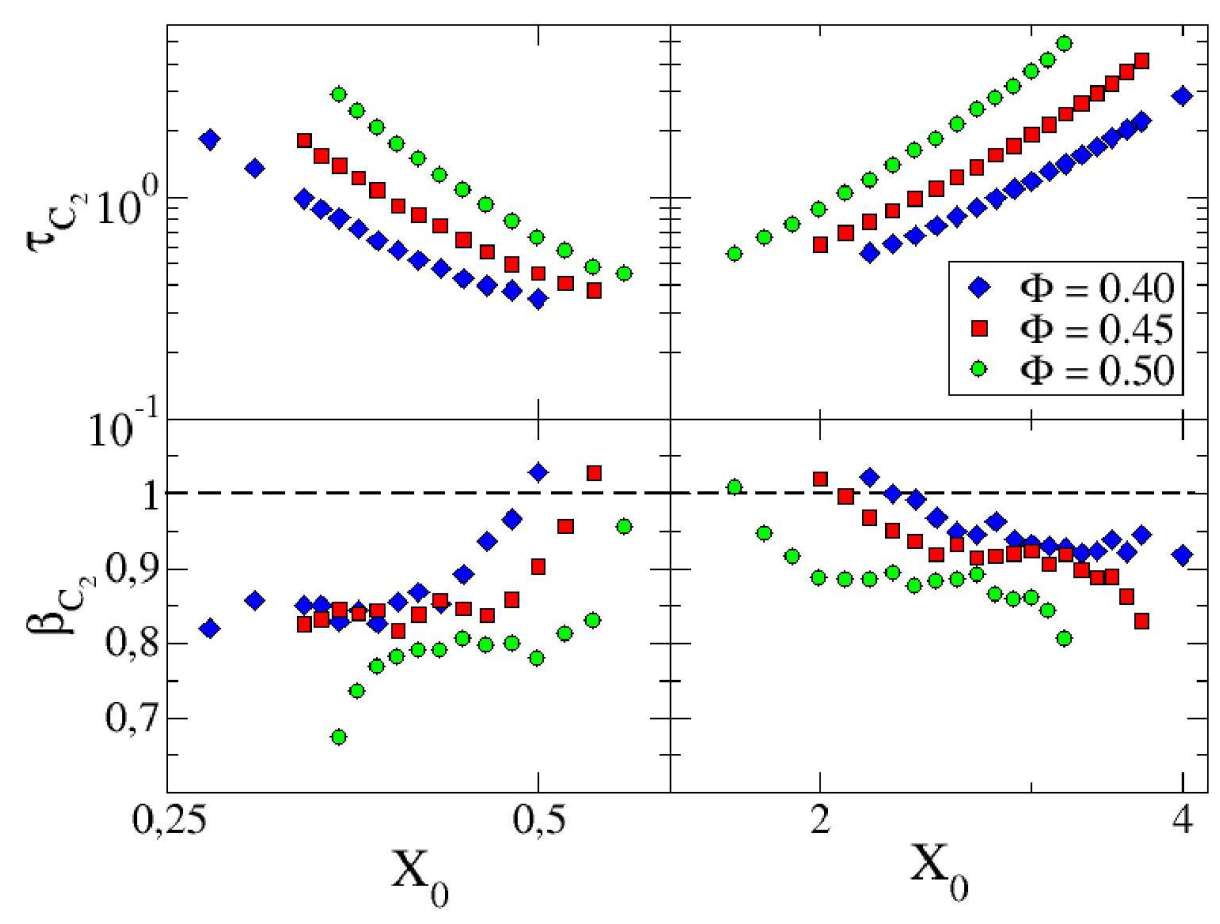

$279 \times 215 \mathrm{~mm}(600 \times 600 \mathrm{DPI})$ 


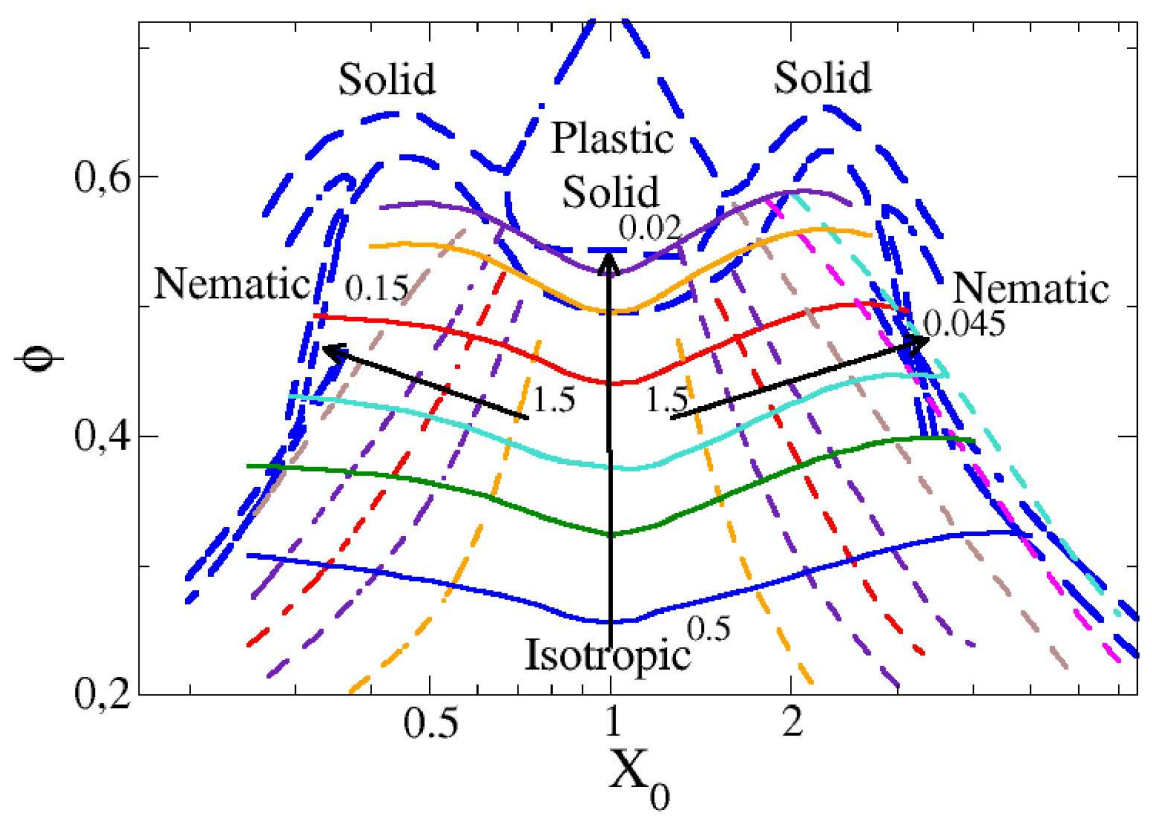

$297 \times 209 m m(600 \times 600$ DPI $)$ 